\title{
Pheophorbide a-mediated photodynamic therapy induces autophagy and apoptosis via the activation of MAPKs in human skin cancer cells
}

\author{
HYO-EUN YOON ${ }^{1}$, SEONE-HEE OH ${ }^{1}$, SOO-A KIM $^{2}$, JUNG-HOON YOON $^{3}$ and SANG-GUN AHN ${ }^{1}$ \\ ${ }^{1}$ Department of Pathology, College of Dentistry, Chosun University, Gwangju 501-759; \\ ${ }^{2}$ Department of Biochemistry, Oriental Medicine, Dongguk University, Gyeongju 780-714; \\ ${ }^{3}$ Department of Oral and Maxillofacial Pathology, College of Dentistry, \\ Wonkwang University, Daejeon 302-120, Republic of Korea
}

Received September 9, 2013; Accepted October 24, 2013

DOI: 10.3892/or.2013.2856

\begin{abstract}
Pheophorbide a $(\mathrm{Pa})$, a chlorophyll derivative, is a photosensitizer that can induce significant antitumor effects in several types of tumor cells. The present study investigated the mechanism of Pa-mediated photodynamic therapy (Pa-PDT) in the human skin cancer cell lines A431 and G361. PDT significantly inhibited the cell growth in a Pa-concentrationdependent manner. We observed increased expression of Beclin-1, LC3B and ATG5, which are markers of autophagy, after PDT treatment in A431 cells but not in G361 cells. In G361 cells, Pa-PDT strongly induced PARP cleavage and subsequent apoptosis, which was confirmed using Annexin V/Propidium iodide double staining. Pa-PDT predominantly exhibited its antitumor effects via activation of ERK1/2 and p38 in A431 and G361 cells, respectively. An in vivo study using the CAM xenograft model demonstrated that Pa-PDT strongly induced autophagy and apoptosis in A431-transplanted tumors and/ or apoptosis in G361-transplanted tumors. These results may provide a basis for understanding the underlying mechanisms of Pa-PDT and for developing Pa-PDT as a therapy for skin cancer.
\end{abstract}

\section{Introduction}

Photodynamic therapy (PDT) is used clinically to treat malignant cancer (1) and is recognized as a minimally invasive therapeutic strategy. PDT leads to the photochemical generation of cytotoxic singlet oxygen through light irradiation of

Correspondence to: Professor Sang-Gun Ahn, Department of Pathology, College of Dentistry, Chosun University, 375 SeosukDong, Dong-gu, Gwangju 501-759, Republic of Korea

E-mail: ahnsg@chosun.ac.kr

Key words: autophagy, apoptosis, pheophorbide a, photodynamic therapy, human skin cancer cells the photosensitizer, which ultimately kills the target cancer cells (2).

The chlorophyll-based photosensitizer, pheophorbide a $(\mathrm{Pa})$, localizes to the mitochondria and exhibits an antitumor effect on human lung cancer, liver cancer and TPA-induced skin tumor in in vivo models (3-5). Furthermore, Pa-mediated PDT (Pa-PDT) leads to the depolarization of mitochondrial membrane potential by the rapid generation of singlet oxygen during light irradiation and inhibits tumor growth in a number of human cancer cells, including Jurkat leukemia, pigmented melanoma, colonic cancer and pancreatic carcinoma cells (6-9). The inhibitory effect of Pa-PDT has also been reported in the hepatitis B virus- and hepatitis $\mathrm{C}$ virus-induced human hepatoma cell lines with multidrug resistance $(10,11)$. Pa-PDT has been reported to induce the release of cytochrome $c$ and trigger the activation of the mitochondrial-mediated apoptosis pathway in malignant carcinoma cells (4,10-13). Although photosensitizer-mediated PDT exerts antitumor effects in several types of cancer, the molecular mechanisms of PDT signaling remain unknown.

The most common types of skin cancer are non-melanoma skin cancers (NMSCs): basal cell carcinoma (BCC) and squamous cell carcinoma (SCC) (14). Clinical studies have applied PDT treatment to NMSC, such as BCC. However, PDT is not commonly used to treat SCC due to the poor penetration of photosensitizers through the keratotic layers generally covering these tumors $(14,15)$. In addition, melanoma skin cancers are also difficult to treat with PDT as, unlike BCC, melanomas grow aggressively, and the highly pigmented melanomas are unaffected by treatment with photosensitizers that absorb in the visible range $(16,17)$.

In the present study, the therapeutic potential of Pa-PDT was evaluated in the human non-melanoma and melanoma skin cancer cell lines, A431 and G361. Our findings provided the first evidence that Pa-PDT can induce autophagy and apoptosis pathways by upregulating ERK1/2 and p38 MAPK activation, and our research demonstrated that Pa-PDT can inhibit the growth of both tumors in vivo, suggesting that Pa-PDT may be an effective alternative therapy to treat skin cancer. 


\section{Materials and methods}

Cell culture and reagents. A human epithelial carcinoma cell line (A431) and malignant melanoma cell line (G361) were maintained in DMEM medium. Both cell lines were cultured in medium supplemented with $10 \%$ fetal bovine serum (FBS), $100 \mathrm{U} / \mathrm{ml}$ of penicillin and $100 \mu \mathrm{g} / \mathrm{ml}$ of streptomycin and maintained at $37^{\circ} \mathrm{C}$ in a humidified incubator with $5 \% \mathrm{CO}_{2}$. $\mathrm{Pa}$ was prepared as a $10 \mathrm{mM}$ stock in dimethyl sulfoxide (DMSO; BDH Merck, Darmstadt, Germany). Further dilutions were made in serum-free DMEM.

Photodynamic therapy. The PDT irradiation light source was a light-emitting diode (LED; 613-645 nm; Philips Luxeon Lumileds, San Jose, CA, USA). The cells ( $1 \times 10^{5} /$ well) were preincubated with $\mathrm{Pa}$ in complete growth medium in the dark for $2 \mathrm{~h}$. For the following experiments, the cells were irradiated at $1.25 \mathrm{~J} / \mathrm{cm}^{2}$. After $24 \mathrm{~h}$ of incubation, the cells were rinsed with phosphate-buffered saline (PBS). For the control group, the cells were incubated in the same medium without $\mathrm{Pa}$ or light.

Cell proliferation assay. The MTT (3-[4,5-dimethylthiazol2-yl]-2,5-diphenyl tetrazolium bromide) assay was used to assess cell proliferation after Pa-PDT treatment. After the medium was removed, the cells were incubated with an MTT solution ( $5 \mathrm{mg} / \mathrm{ml}$ in PBS) for $3 \mathrm{~h}$, and the absorbance was measured using an auto ELISA plate reader at $570 \mathrm{~nm}$. In addition, we performed cell proliferation assays using a cell proliferation ELISA kit (Roche Applied Science, Indianapolis, IN, USA).

Western blotting. The cells were treated with Pa-PDT for $24 \mathrm{~h}$. The cells were then washed with PBS and harvested in lysis buffer. Samples containing equal amounts of protein were loaded onto each lane of an SDS-polyacrylamide gel for electrophoresis and subsequently transferred onto a polyvinylidene difluoride membrane. The membranes were blocked and then incubated with antibodies. Antibodies against Beclin-1, Bcl-2, Atg5, LC3B and p-mTOR were purchased from Cell Signaling Technology (Beverly, MA, USA); p-Akt, caspase-7, PARP and $\beta$-actin were purchased from Santa Cruz Biotechnology (Santa Cruz, CA, USA).

Detection and quantification of acidic vesicular organelles with acridine orange staining using flow cytometry. Autophagy is characterized by the formation of acidic vesicular organelles (AVOs) (17). A431 and G361 cells were seeded in $6-\mathrm{cm}^{2}$ plates and incubated for $24 \mathrm{~h}$. After treatment with Pa-PDT for $24 \mathrm{~h}$, acridine orange $(1 \mu \mathrm{g} / \mathrm{ml})$ was added to the living cells for $30 \mathrm{~min}$, and the cells were removed from the plate with trypsin-EDTA and collected in phenol red-free growth medium. Green $(510-530 \mathrm{~nm})$ and red $(650 \mathrm{~nm})$ fluorescence emission from $1 \times 10^{4}$ cells illuminated with blue $(488 \mathrm{~nm})$ excitation light was measured with a FACSCalibur using CellQuest software (Becton-Dickinson).

MDC staining. To observe autophagy formation, skin cells were grown on glass coverslips for $24 \mathrm{~h}$ in a humidified incubator in $5 \% \mathrm{CO}_{2}$ and at $37^{\circ} \mathrm{C}$. After treatment with Pa-PDT for $24 \mathrm{~h}$, the cells were treated with $0.05 \mathrm{mM}$ monodansylcadaverine (MDC; Sigma-Aldrich Chemical) at $37^{\circ} \mathrm{C}$ in $5 \%$
$\mathrm{CO}_{2}$ for $10 \mathrm{~min}$. The cells were then fixed with $4 \%$ paraformaldehyde in PBS for 10 min. Following incubation, the cells were washed three times with PBS and immediately analyzed under a fluorescence microscope (IX-71; Olympus, Tokyo, Japan). Fluorescence of MDC was measured at the excitation wavelength of $380 \mathrm{~nm}$ with an emission filter at $530 \mathrm{~nm}$.

Annexin V-FITC/PI double staining. The cells were harvested and fixed with $70 \%$ ethanol for $1 \mathrm{~h}$ at $4{ }^{\circ} \mathrm{C}$ for cell cycle analysis. After washing with cold PBS, the cells were incubated with DNase-free RNase and propidium iodide (PI) at $37^{\circ} \mathrm{C}$ for $30 \mathrm{~min}$. The specific binding of Annexin V-FITC/PI was performed by incubating the cells for $15 \mathrm{~min}$ at room temperature in a binding buffer (10 mM HEPES, $140 \mathrm{mM} \mathrm{NaCl}$, $2.5 \mathrm{mM} \mathrm{CaCl}_{2}, \mathrm{pH}$ 7.4) containing saturating concentrations of Annexin V-FITC and PI. Following incubation, the cells were pelleted and analyzed in a FACScan analyzer (Beckman Coulter Inc., Fullerton, CA, USA).

Caspase-3 activity assay. Caspase-3 activity was assessed using a caspase- 3 colorimetric assay kit (Clontech, Palo Alto, CA, USA) following the manufacturer's instructions. The cells seeded in 6 -well plates $\left(2 \times 10^{5}\right.$ cells/well) were treated with or without Pa-PDT for $24 \mathrm{~h}$ as previously described. The cells were collected and resuspended in lysis buffer containing $50 \mathrm{mM}$ HEPES, pH 7.4, 0.1\% CHAPS, $1 \mathrm{mM}$ DTT, $0.1 \mathrm{mM}$ EDTA and $0.1 \%$ Triton X-100. Cell lysates were centrifuged at $12,000 \mathrm{x} \mathrm{g}$ for $10 \mathrm{~min}$ at $4^{\circ} \mathrm{C}$. The supernatants were incubated with the reaction buffer containing $2 \mathrm{mM}$ Ac-DEVD-pNA for $1 \mathrm{~h}$ at $37^{\circ} \mathrm{C}$. Caspase activity was determined by measuring the absorbance at $405 \mathrm{~nm}$.

In vivo chorioallantoic membrane assay (CAM) assay and immunohistochemistry assay. The CAM assay was used to examine the inhibition of tumor growth in vivo. The CAM assay was performed as previously described (18). Briefly, fertilized chicken eggs were transferred to an egg incubator maintained at $37^{\circ} \mathrm{C}$ and $50 \%$ humidity and allowed to grow for 10 days. The fertilized chick eggs were sterilized, and a $1-\mathrm{cm}^{2}$ window was cut, using the false air sac technique, on one side of the egg to expose the CAM. Skin cancer cells $\left(2 \times 10^{6}\right)$ were placed on the exposed CAM, and the windows were sealed with transparent tape. The eggs were incubated in a humidified incubator at $37^{\circ} \mathrm{C}$ for three days and pre-treated with normal saline or Pa $(0.1 \mu \mathrm{M})$ prior to light exposure $\left(1.25 \mathrm{~J} / \mathrm{cm}^{2}\right)$. At the next day, the excised tumors were fixed in $10 \%$ formalin and paraffin-embedded and cell staining was performed with hematoxylin and eosin (H\&E).

Statistical analysis. The statistical analyses were performed with data obtained from three independent experiments. The data are represented as the mean \pm SEM. A P-value $<0.05$ was considered to indicate a statistically significant result.

\section{Results}

Antitumor effect of Pa-PDT on human skin cancer cells. To test the effect of Pa-PDT, A431 and G361 cells were pre-treated with different concentrations of $\mathrm{Pa}(0.05$ or $0.1 \mu \mathrm{M})$ for $2 \mathrm{~h}$ in the dark, followed by photoactivation with $1.25 \mathrm{~J} / \mathrm{cm}^{2}$ of LED. 

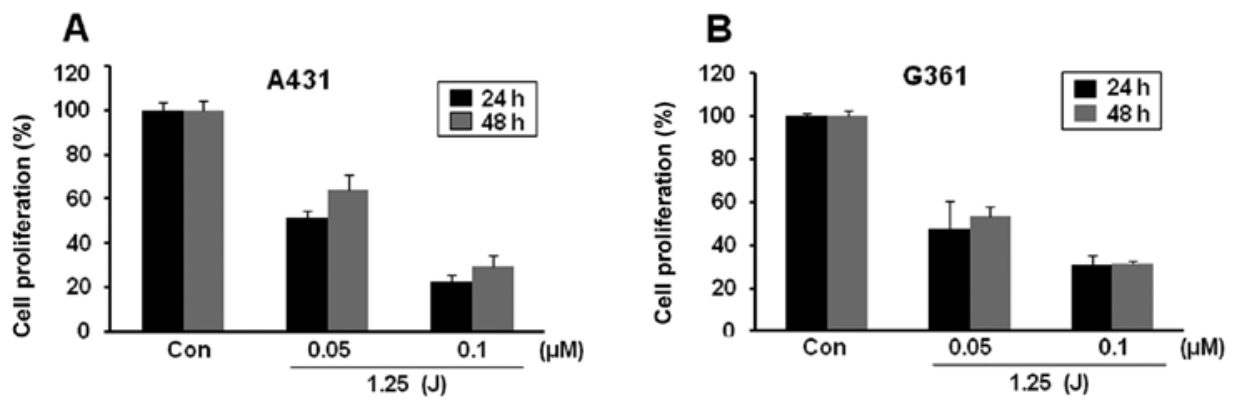

Figure 1. The effects of Pa-PDT on human skin cancer cells. (A and B) A431 and G361 cells were preincubated with different Pa concentrations (0.05 and $0.1 \mu \mathrm{M})$ for $2 \mathrm{~h}$ and then illuminated $\left(1.25 \mathrm{~J} / \mathrm{cm}^{2}\right)$. Cell proliferation was measured using an MTT assay at 24 or $48 \mathrm{~h}$ after Pa-PDT treatment.
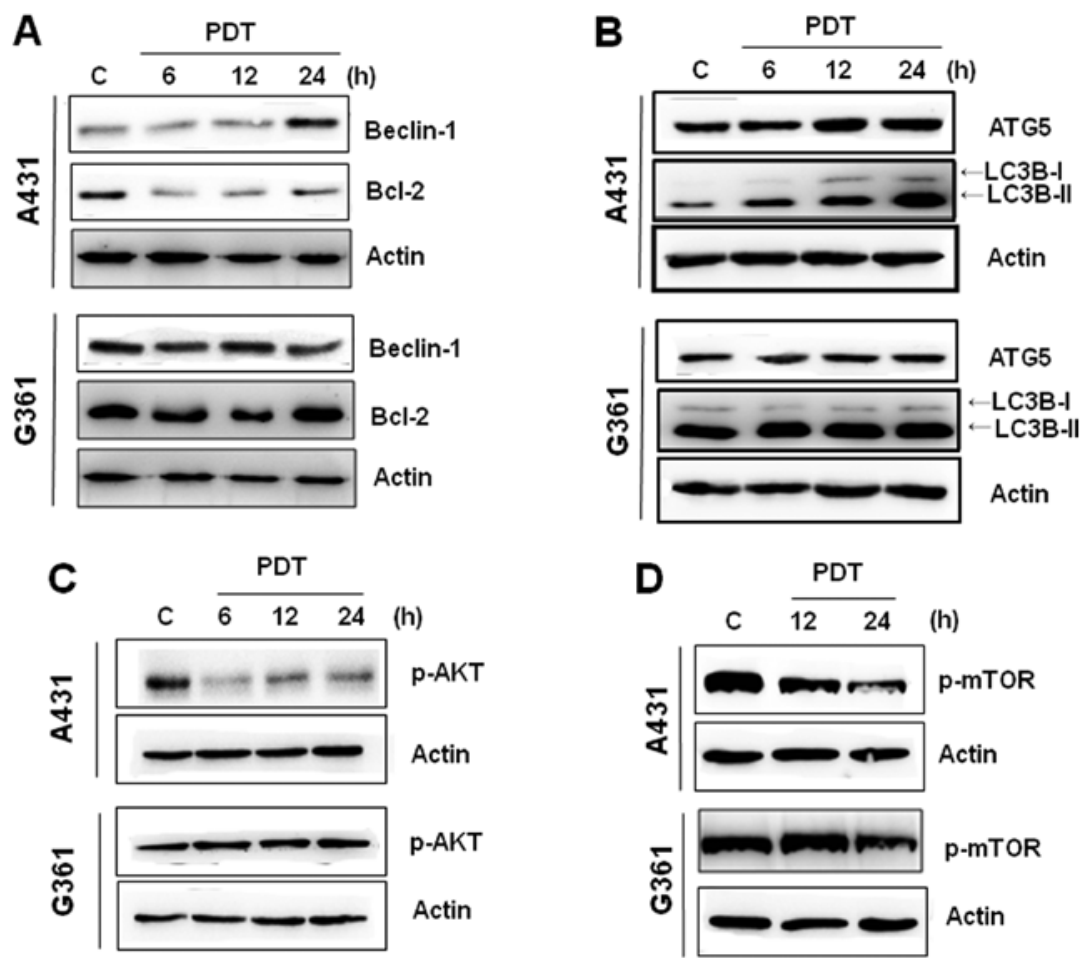

Figure 2. Induction of autophagy in Pa-PDT-treated cells. (A) The expression of Beclin-1 and Bcl-2 by Pa-PDT. A431 and G361 cells were treated with $0.05 \mu \mathrm{M}$ Pa with illumination and subjected to western blotting. (B) Time-dependent detection of LC3B and ATG-5 expression by western blotting of whole cell lysates of human A431 and G361 cells after PDT treatment. (C and D) The regulation of Akt and mTOR phosphorylation after Pa-PDT treatment. The cells were treated with $0.05 \mu \mathrm{M}$ Pa-PDT for the indicated time periods and subjected to western blotting. Actin was used as a loading control.

Twenty-four hours after exposure, the cell proliferation was determined using an MTT assay. We found that these cell lines exhibited no significant decrease in cell proliferation due to $\mathrm{Pa}$ or light alone (data not shown). However, the cell proliferation in both cell lines was severely decreased by Pa-PDT treatment. In A431 cells, $\mathrm{Pa}$ doses of 0.05 and $0.1 \mu \mathrm{M}$ with light resulted in cell growth inhibition rates of 48.8 and $77.7 \%$ at $24 \mathrm{~h}$, respectively (Fig. 1A). Similarly, Pa-PDT also induced a significant cytotoxicity in G361 melanoma cells in a Pa-dosedependent manner (Fig. 1B). After $48 \mathrm{~h}$ PDT treatment, the cell growth slightly recovered in both cells compared with growth at $24 \mathrm{~h}$ (Fig. 1). These results demonstrate that Pa-PDT exerts an antiproliferative effect on human skin cancer cells.

Expression of LC $3 B$ and Beclin 1 in Pa-PDT-treated cells. To further elucidate the underlying mechanisms of Pa-PDTinduced cell growth inhibition, we assessed the levels of
Beclin 1, Bcl-2, ATG5 and LC3B-I/II, which play a crucial role in autophagy. Western blotting revealed that Pa-PDT induced an increase in the expressions of Beclin 1 in a time-dependent manner, whereas the expression of $\mathrm{Bcl}-2$ (Beclin 1 regulatory proteins) was reduced in A431 cells (Fig. 2A). The induction of ATG5 and LC3-II expression began to be clearly observed at $12 \mathrm{~h}$ after Pa-PDT treatment (Fig. 2B). However, the G361 cell line exhibited no significant expression of these proteins after Pa-PDT treatment. This finding suggested the possibility that the mechanism of Pa-PDT-mediated cell growth inhibition in A431 cells, but not in G361 cells, was related to the Beclin 1-dependent autophagy.

The mTOR/AKT pathway is a major signaling pathway that regulates autophagy (19). To confirm whether Pa-PDT regulated autophagy in A431 cells, we assessed the influence of Pa-PDT on the activation of mTOR and Akt. In treating A431 cells with Pa-PDT, the levels of p-AKT and p-mTOR 

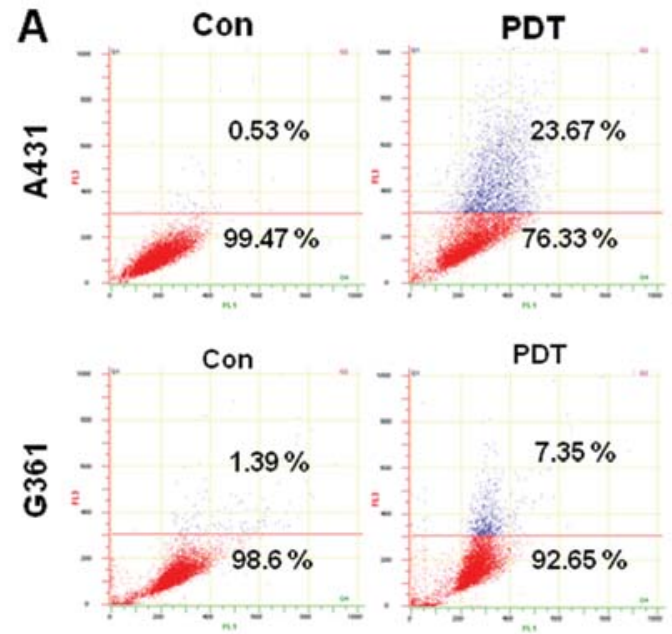

B
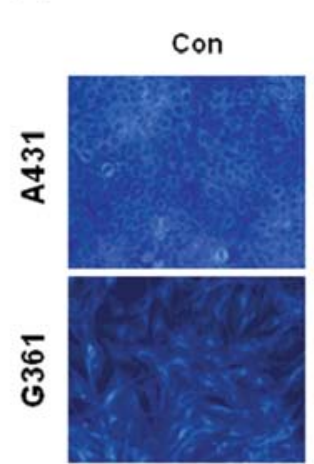

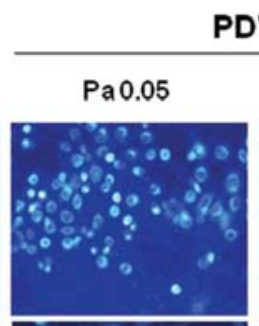

PDT

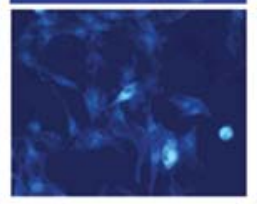

\section{$\mathrm{Pa} 0.1$}
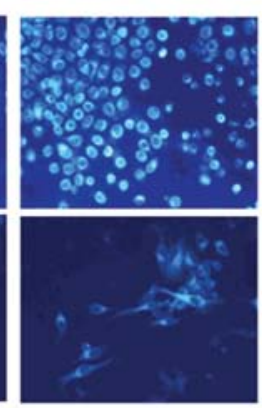

Figure 3. Autophagic cell death induced by Pa-PDT in A431 cells. (A) After treatment with Pa-PDT, acridine orange (1 $\mu$ g/ml) was added to the living cells for $30 \mathrm{~min}$. Green $(510-530 \mathrm{~nm})$ and red $(650 \mathrm{~nm})$ fluorescence emission from $1 \times 10^{4}$ cells illuminated with blue (488 $\left.\mathrm{nm}\right)$ excitation light was measured with a FACSCalibur flow cytometer. The representative results from three independent experiments are shown. (B) Photomicrographs of MDC staining. Pa-PDTtreated cells were photographed at x 100 magnification. The cells were pretreated with the indicated concentration of Pa for $2 \mathrm{~h}$ and subjected to LED. The cells were incubated with $\mathrm{MDC}(50 \mu \mathrm{M})$ for $10 \mathrm{~min}$ and observed under a fluorescence microscope.

decreased at 6 and $12 \mathrm{~h}$ (Fig. 2C and D). In G361 cells, Pa-PDT did not affect the expression of p-Akt but slightly decreased the level of phospho-mTOR at $24 \mathrm{~h}$. These results indicate that Pa-PDT inhibits the Akt/mTOR pathway and that these changes induce autophagy in A431 cells.

Quantization and detection of autophagic vacuoles by $P a-P D T$. Autophagy is characterized by the formation of acidic AVOs (20). To confirm the presence of vacuoles in Pa-PDT-treated A431 cells, we performed acridine orange staining to obtain acidic AVOs. As shown in Fig. 3A, the number of AVOs was increased in A431 cells treated with Pa-PDT compared with the control cells. In G361 cells, the number of AVOs was slightly increased. Similar results were also obtained by MDC staining. The fluorescent compound MDC is a specific marker for autolysosomes and is commonly used to stain autophagic vesicles. A431 and G361 cells were treated with PDT for $24 \mathrm{~h}$ and analyzed using fluorescence microscopy. As shown in Fig. 3B, in the control cells, MDC-labeled vacuoles were not detected. However, in Pa-PDT-treated A431 cells, MDC-labeled cells were strongly detected, and the number of these cells increased during treatment in a concentration-dependent manner. In G361 cells, MDC-labeled cells were weakly detected. Collectively, these results also suggest that Pa-PDT induced autophagy in A431 cells but not in G361 cells.

Activation of ERK1/2 and p38 MAPK in Pa-PDT-treated cells. It has been suggested that PDT can activate the MAPK pathway and regulate the cell death process $(21,22)$. The activation of MAPKs in Pa-PDT-treated cells was monitored by western blotting. As shown in Fig. 4A, the phosphorylation of ERK1/2 and p38 MAPK was induced after $6 \mathrm{~h}$ of Pa-PDT treatment in A431 cells, whereas the activation of JNK was not detected. Similarly, the LC3B-I/II level was increased in a time-dependent manner during Pa-PDT treatment. In G361 cells, the phosphorylation of p38 MAPK was induced at $24 \mathrm{~h}$ after Pa-PDT treatment, whereas the phosphorylation of ERK was slightly increased at $12 \mathrm{~h}$ (Fig. 4A).

We next examined whether MAPK signaling was involved in Pa-PDT-induced autophagy. A431 cells were treated with an ERK1/2 inhibitor (U0126) in a gradient for $6 \mathrm{~h}$ followed by western blot analysis. As expected, ERK phosphorylation by PDT was inhibited by treatment with U0126 in a time-dependent manner. Following U0126 treatment, LC3B-II protein levels decreased in A431 cells, suggesting that ERK1/2 signaling was involved in Pa-PDT-induced autophagy (Fig. 4B). However, the inhibition of ERK phosphorylation by U0126 did not affect the LC3-II levels in G361 cells, suggesting that Pa-PDT induced cell growth inhibition by other pathways in G361 cells. To confirm the effect of U0126 on Pa-PDT-induced autophagy, we performed acridine orange staining to visualize autophagy using fluorescence microscopy. As shown in Fig. 4C, the control cells primarily emitted green fluorescence, indicating a lack of AVO. Pa-PDT strongly increased the intensity of red fluorescence in A431 cells but not in G361 cells. Alternatively, treatment of cells with $20 \mu \mathrm{M}$ U0126 decreased the formation of Pa-PDT-induced AVO. These results indicate that the ERK1/2 pathway is involved in Pa-PDT-induced autophagy in A431 cells.

To further examine the role of p38 MAPK in Pa-PDTmediated cytotoxicity, the p38 MAPK-specific inhibitor SB202190 was used. Pa-PDT induced PARP cleavage in A431 and G361 cells. SB202190 inhibited PARP cleavage in both cells. However, U0126 did not block PARP cleavage by Pa-PDT (Fig. 4D). Collectively, these results suggest that Pa-PDT also leads to apoptosis through p38 MAPK activation in A431 and G361 cells.

The effects of Pa-PDT on apoptosis. To determine whether the decrease in viability of A431 and G361 cells was also caused by the induction of apoptosis, we quantified apoptosis by flow cytometry using the Annexin V-FITC/PI double staining assay. As shown in Fig. 5A, a significant number of apoptotic 

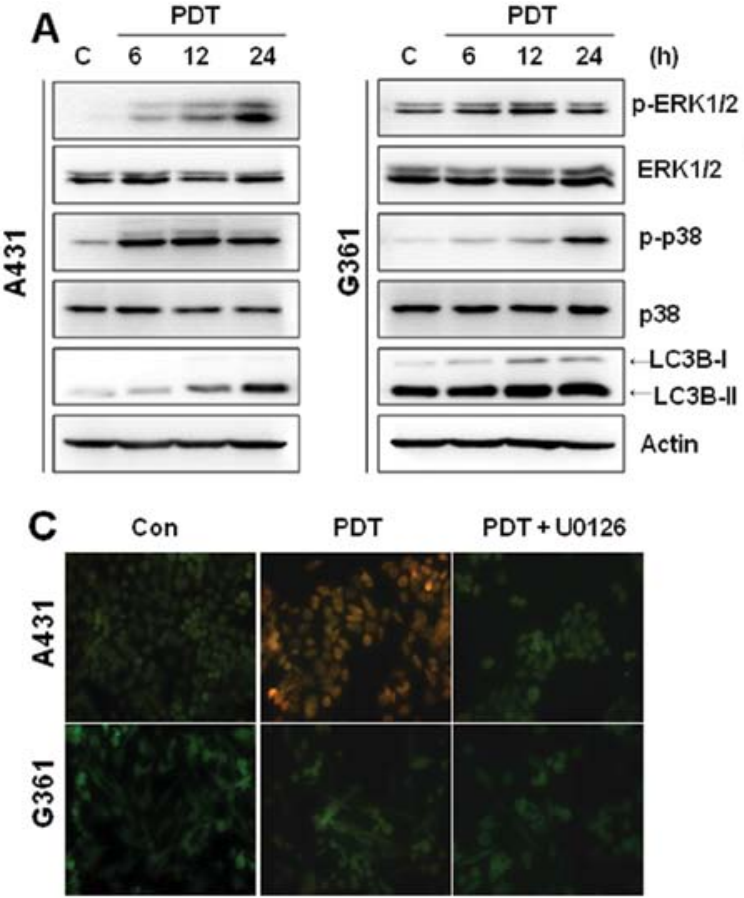

B
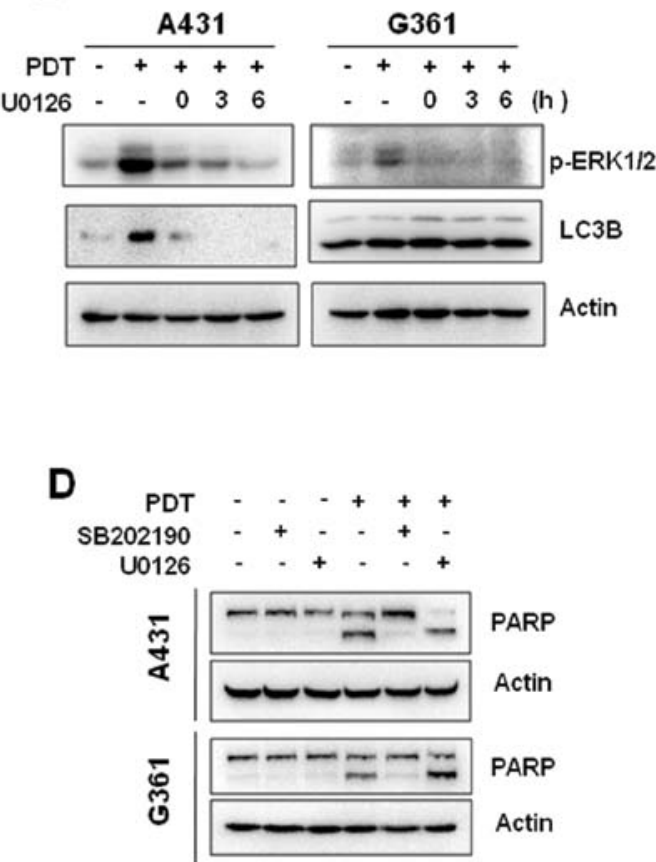

Figure 4. Pa-PDT activated ERK1/2 and/or p38 MAPK in A431 and G361 cells. (A) Western blot analysis of ERK and p38 MAPK activation. The cells were treated with Pa-PDT for the indicated time periods. The levels of ERK1/2, p38 and LC3B-I/II were measured by western blotting. (B) The effect of ERK inhibitors on LC3B expression. The cells were treated with $20 \mu \mathrm{M}$ U0126 for the indicated time periods. The phosphorylated ERK1/2 protein was analyzed. (C) The effect of U0126 on Pa-PDT-induced acidic vesicular organelle (AVO) formation. After Pa-PDT treatment with U0126, acridine orange (1 $\mu \mathrm{g} / \mathrm{ml}) \mathrm{was}$ added to the living cells for $30 \mathrm{~min}$ and the cells were visualized under a fluorescence microscope. (D) The effect of p38 inhibitors on PARP activation. The cells were treated with Pa-PDT and $20 \mu \mathrm{M}$ SB202190 or $20 \mu \mathrm{M}$ U0126; subsequently, intracellular PARP cleavage was determined by western blot analysis.
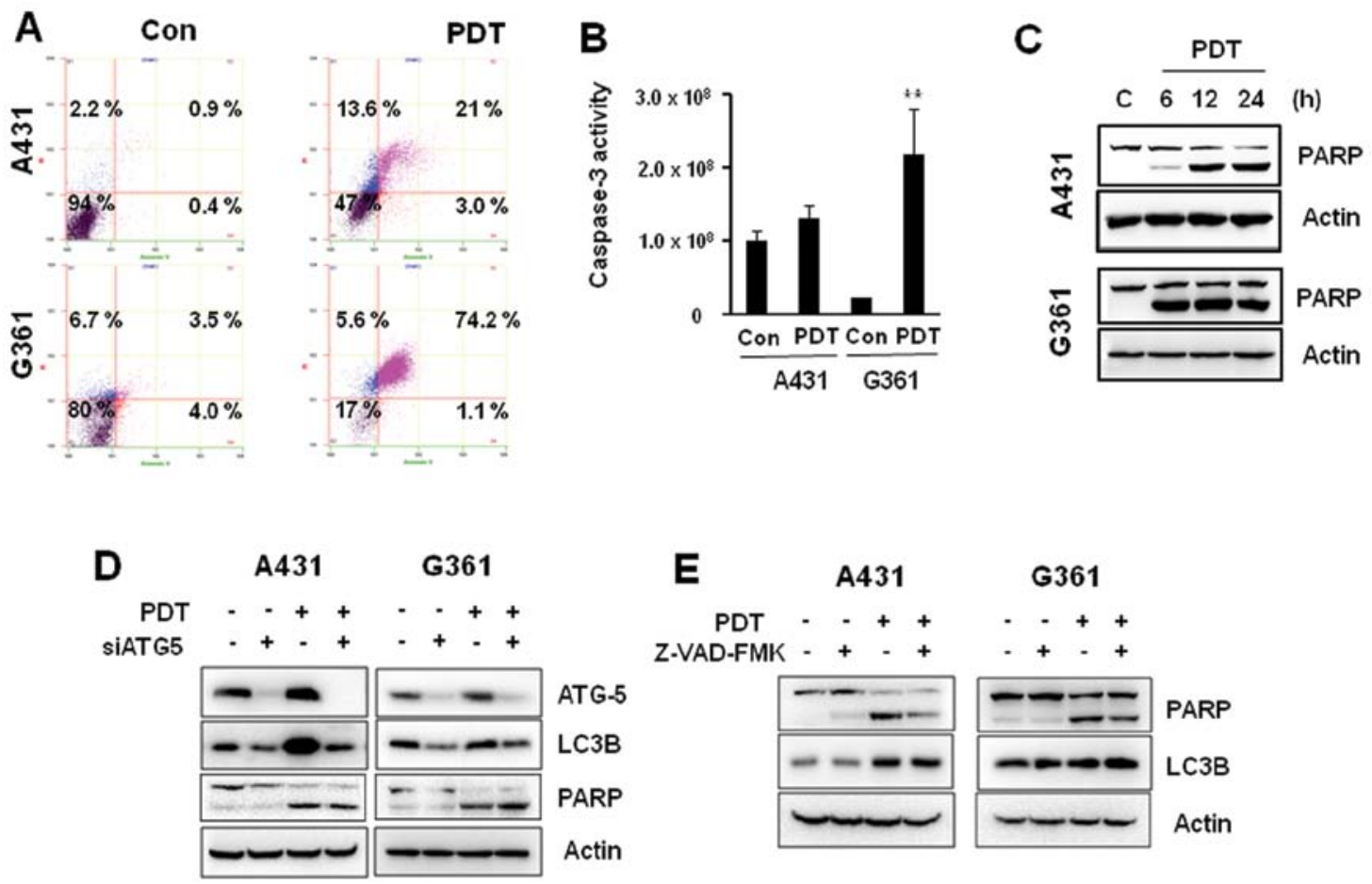

\section{E}

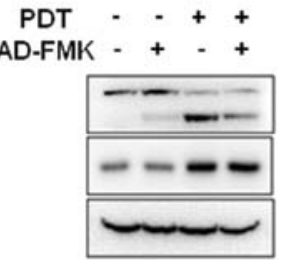

G361

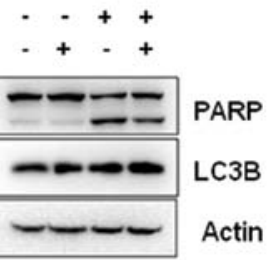

Figure 5. Pa-PDT induces apoptosis in A431 and G361 cells. (A) The quantification of apoptotic cells using flow cytometric analysis. A431 and G361 cells were treated with $0.05 \mu \mathrm{M} \mathrm{Pa}$ for $2 \mathrm{~h}$ and irradiated with $1.25 \mathrm{~J} / \mathrm{cm}^{2}$, respectively. The cells were harvested and stained with Annexin V-FITC/PI, followed by flow cytometric analysis. (B) The effect of Pa-PDT on caspase-3 activity. The activity of caspase-3 in cells was measured using a caspase-3 enzyme colorimetric assay kit. Each value is expressed as the mean \pm SD based on three independent experiments. (C) Western blot analysis of PARP activation after Pa-PDT treatment. The cells were treated with Pa-PDT and cell lysates were subjected to western blot analysis using antibodies against cleaved PARP. (D) The modulation of Pa-PDT-induced apoptosis by knockdown of ATG-5. The cells were transfected with either ATG-5 siRNA or a non-silencing control siRNA for $24 \mathrm{~h}$ and subsequently treated with Pa-PDT. The levels of ATG-5, LC3B and PARP were measured by western blotting. (E) The effect of apoptosis inhibition on Pa-PDT-induced autophagy in A431 cells. The cells were treated with either $20 \mu \mathrm{M}$ of the pan-caspase inhibitor Z-VAD-FMK or control for $2 \mathrm{~h}$ and treated with Pa-PDT before measuring PARP cleavage and LC3B expression by western blotting. 
A

A431

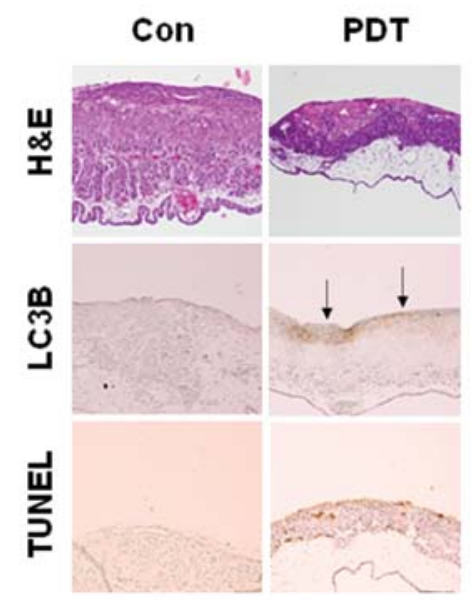

G361

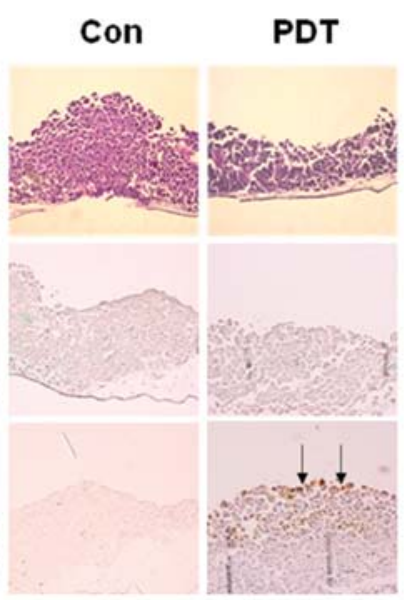

B

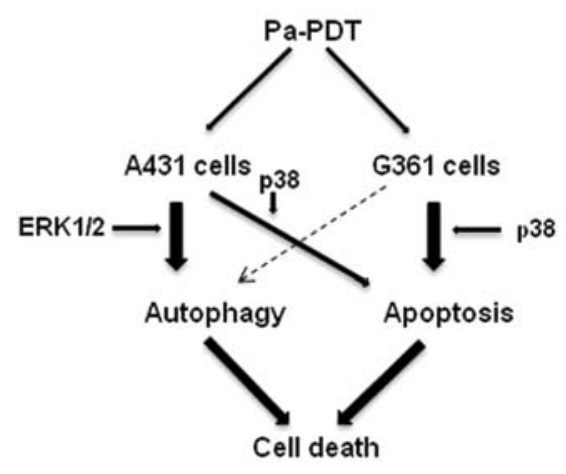

Figure 6. The effect of Pa-PDT on tumor formation in in vivo CAM assay. (A) In vivo CAM assay by Pa-PDT. The fertilized eggs were implanted with A431 and G361 cells $\left(2 \times 10^{6}\right.$ cells) for 3 days and incubated in a humidified incubator at $37^{\circ} \mathrm{C}$ for an additional $24 \mathrm{~h}$ after Pa-PDT treatment. Hematoxylin and eosin (H\&E) staining and immunohistochemistry with anti-LC3B antibody were performed on paraffin sections from the tumors. (B) Schematic model of cell death regulated by Pa-PDT in skin cancer cells.

cells (74.2\%) was detected in G361 cells after Pa-PDT treatment, whereas $<24 \%$ apoptotic-positive cells were detected in A431 cells. Consistent with this observation, caspase- 3 activity was also strongly detected in G361 cells treated with Pa-PDT compared to A431 cells (Fig. 5B). In addition, the cleaved form of PARP was increased by Pa-PDT treatment in a timedependent manner in both cells. However, PARP cleavage was stronger in G361 cells than in A431 cells (Fig. 5C).

To investigate potential cross-talk between autophagy and apoptosis induction in response to Pa-PDT, we investigated whether Pa-PDT-induced apoptosis could be inhibited by siRNA against ATG-5. In A431 cells, Pa-PDT-induced LC3B expression was inhibited by ATG-5 siRNA (Fig. 5D). However, Pa-PDT-induced PARP cleavage was not inhibited by ATG-5 siRNA in A431 and G361 cells. In addition, 3MA, an early stage inhibitor of autophagy, did not block Pa-PDT-induced PARP cleavage (data not shown), suggesting that autophagy is unrelated to Pa-PDT-mediated apoptosis.

Additionally, to determine whether Pa-PDT-induced apoptosis affected the autophagy pathway, we examined the expression levels of key autophagy proteins, LC3B, during treatment with the caspase inhibitor zVAD-fmk. Pretreatment of cells with the caspase inhibitor zVAD-fmk did not prevent Pa-PDT-induced expression of LC3B proteins in A431 cells (Fig. 5E). Our data demonstrated that Pa-PDT induces autophagy and/or apoptosis in A431 and G361 cells independently.

The effect of Pa-PDT on tumor growth and cell death in a skin cancer cell-xenograft CAM model. To identify the pathobiological characteristics of the transplantation tumors in the CAM, hematoxylin-eosin (H\&E) staining and immunohistochemical analysis were performed.

Histological examination demonstrated that Pa-PDT reduced tumor thickness and increased cell death in both cell-xenograft CAM. Specifically, the expression of LC3B protein was more strongly increased by Pa-PDT in A431- implanted tumors. Additionally, TUNEL staining confirmed that TUNEL-positive cells were more frequently observed in G361-implanted tumor sections (Fig. 6A). These results demonstrate that Pa-PDT leads to selective and effective cell death of skin cancer cells through the autophagy and apoptosis pathway.

\section{Discussion}

PDT is used worldwide not only in the primary treatment for malignant and premalignant skin cancer but also for adjuvant treatment in lung, brain, esophageal, biliary and urinary tract cancer (2,14-16). The rapid generation of ROS observed in PDT-treated cells has been reported to induce damage to mitochondria or the endoplasmic reticulum (ER) and lead to apoptosis $(23,24)$. Although apoptosis has been reported as the predominant cell death modality after photosensitizermediated PDT, the mechanism of PDT-mediated cell death is largely unknown.

The present study was designed to determine whether Pa-PDT exhibits anticancer properties in non-melanoma A431 and melanoma G361 skin cancer cells and to further investigate the underlying mechanisms of Pa-PDT-induced cell death. We observed that Pa-PDT predominantly induced autophagy in A431 cells, as indicated by multiple independent approaches that either revealed the expression of autophagy-specific proteins or the formation of autophagic vacuoles. Pa-PDT induced Beclin 1-dependent autophagy through ERK1/2 activation. By contrast, Pa-PDT mainly induced the apoptotic pathway through p38 MAPK activation in G361 cells.

Previous studies demonstrated that the Akt/mTOR pathway is the major pathway that regulates autophagy (25). The inhibition of Akt phosphorylation and downstream mTOR signaling contribute to the initiation of autophagy $(26,27)$. We clearly demonstrated that Pa-PDT treatment inhibits the phosphorylation of Akt and mTOR in A431 cells but not in G361 cells. These results demonstrated that Pa-PDT-induced autophagy is 
mediated by the downregulation of Akt/mTOR in A431 cells. In particular, the phosphorylation of ERK1/2 and p38 MAPK was induced by Pa-PDT in A431 cells. In G361 cells, the activation of p38 MAPK was observed, whereas the phosphorylation of ERK1/2 was not markedly induced by Pa-PDT. From these results, we considered Pa-PDT-induced cell death to be related to the p38 and/or ERK1/2 pathway in skin cancer cells. The inhibition of ERK activity by the ERK inhibitor U0126 reduced vesicle formation and LC3B expression in Pa-PDT treated A431 cells. These results suggest that ERK1/2 activation could mediate autophagy in Pa-PDT-treated A431 cells.

In addition, we observed PARP cleavage in Pa-PDT-treated A431 and/or G361 cells. Notably,PARP cleavage was suppressed by the p38 inhibitor, SB202190. The findings supported the induction of p38-mediated apoptosis during Pa-PDT treatment. The reason for the difference between A361 and G361 cell responses to Pa-PDT treatment is unclear. However, the mechanism of action of PDT might depend on the subcellular localization and molecular targets of the photosensitizer, the metabolic potential and the genotype of the tumor cell type.

It has been suggested that the autophagic response observed in cells treated with diverse cytotoxic agents is involved in protecting cells from apoptosis or, alternatively, is associated with a mechanism contributing to apoptosis (28-30). Despite these studies, the relationship between autophagy and apoptosis remains poorly understood.

We investigated whether Pa-PDT-induced autophagy leads to the activation of the apoptotic pathway. A431 and G361 cells were treated with Pa-PDT in the presence or absence of 3-MA and chloroquine (CQ), specific inhibitors of the early and latestage autophagic process, respectively. 3-MA and CQ did not inhibit the activation of PARP induced by Pa-PDT in either cell line (data not shown). Similarly, the suppression of ATG-5 using siRNA did not block Pa-PDT-induced PARP cleavage. In addition, caspase inhibition did not inhibit Pa-PDT-induced autophagy-related protein expression in A431 cells. These findings suggest that the interplay between Pa-PDT-induced autophagy and apoptosis does not exist in A431 and G361 cells.

Our previous study demonstrated the therapeutic potential of Pa-PDT on YD-10B cells as a model of human oral cancer. Our findings revealed that autophagy contributes to Pa-PDTmediated cell growth inhibition. In that study, Pa-PDT reduced the phosphorylation of ERK, whereas the phosphorylation of p38 and JNK were unchanged (31). Therefore, the Pa-PDTinduced MAPK regulation may trigger different intracellular cell death pathways in a cell type-specific manner.

In conclusion, we demonstrated for the first time that Pa-PDT induces autophagy and apoptosis in A431 cells. Pa-PDT-induced cell death is carried out through ERK1/2mediated autophagy as well as apoptosis secondary to p38 MAPK activation. Alternatively, the potent antitumor effect of Pa-PDT on G361 cells was induced via p38-mediated caspase-3-dependent apoptotic pathways. Therefore, Pa-PDT is a potential therapy for human skin cancer and induces multiple death pathways via MAPK activation.

\section{Acknowledgements}

We thank Dr Se-Won Park for insightful discussion. This study was supported by the National Research Foundation of Korea (NRF) funded by the Ministry of Science, ICT \& Future Planning (No. R13-2008-010-00000-0). Dr J.-H. Yoon was supported by a grant of the Korean Health Technology R\&D Project, Ministry for Health, Welfare \& Family Affairs, Republic of Korea (No. A100490).

\section{References}

1. Chen J, Keltner L, Christophersen J, Zheng F, Krouse M, Singhal A and Wang SS: New technology for deep light distribution in tissue for phototherapy. Cancer J 8: 154-163, 2002.

2. Dolmans DE, Fukumura D and Jain R: Photodynamic therapy for cancer. Nat Rev Cancer 3: 380-387, 2003.

3. Yin X, Zhou J, Jie C, Xing D and Zhang Y: Anticancer activity and mechanism of Scutellaria barbata extract on human lung cancer cell line A549. Life Sci 75: 2233-2244, 2004.

4. Chan JY, Tang PM, Hon PM, Au SW, Tsui SK, Waye MM, Kong SK, Mak TC and Fung KP: Pheophorbide a, a major antitumor component purified from Scutellaria barbata, induces apoptosis in human hepatocellular carcinoma cells. Planta Med 72: 28-33, 2006.

5. Nakamura Y, Murakami A, Koshimizu K and Ohigashi H: Inhibitory effect of pheophorbide a, a chlorophyll-related compound, on skin tumor promotion in ICR mouse. Cancer Lett 108: 247-255, 1996.

6. Lee WY, Lim DS, Ko SH, Park YJ, Ryu KS, Ahn MY, Kim YR, Lee DW and Cho CW: Photoactivation of pheophorbide a induces a mitochondrial mediated apoptosis in Jurkat leukaemia cells. J Photochem Photobiol B 75: 119-126, 2004.

7. Hajri A, Wack S, Meyer C, Smith MK, Leberquier C, Kedinger M and Aprahamian M: In vitro and in vivo efficacy of photofrin and pheophorbide a, a bacteriochlorin, in photodynamic therapy of colonic cancer cells. Photochem Photobiol 75: 140-148, 2002.

8. Jin ZH, Miyoshi N, Ishiguro K, Umemura S, Kawabata K, Yumita N, Sakata I, Takaoka K, Udagawa T, Nakajima S, Tajiri H, Ueda K, Fukuda M and Kumakiri M: Combination effect of photodynamic and sonodynamic therapy on experimental skin squamous cell carcinoma in $\mathrm{C} 3 \mathrm{H} / \mathrm{HeN}$ mice. J Dermatol 27: 294-306, 2000

9. Hajri A, Coffy S, Vallat F, Evrard S, Marescaux J and Aprahamian M: Human pancreatic carcinoma cells are sensitive to photodynamic therapy in vitro and in vivo. Br J Surg 86: 899-906, 1999.

10. Tang PM, Chan JY, Au SW, Kong SK, Tsui SK, Waye MM, Mak TC, Fong WP and Fung KP: Pheophorbide a, an active compound isolated from Scutellaria barbata, possesses photodynamic activities by inducing apoptosis in human hepatocellular carcinoma. Cancer Biol Ther 5: 1111-1116, 2006.

11. Tang PM, Zhang DM, Xuan NH, Tsui SK, Waye MM, Kong SK, Fong WP and Fung KP: Photodynamic therapy inhibits P-glycoprotein mediated multidrug resistance via JNK activation in human hepatocellular carcinoma using the photosensitizer pheophorbide a. Mol Cancer 8: 56-66, 2009.

12. Tang PM, Liu XZ, Zhang DM, Fong WP and Fung KP: Pheophorbide a based photodynamic therapy induces apoptosis via mitochondrial-mediated pathway in human uterine carcinosarcoma. Cancer Biol Ther 8: 533-539, 2009.

13. Ahn MY, Kwon SM, Kim YC, Ahn SG and Yoon JH: Pheophorbide a-mediated photodynamic therapy induces apoptotic cell death in murine oral squamous cell carcinoma in vitro and in vivo. Oncol Rep 27: 1772-1778, 2012.

14. Choudhary S, Nouri K and Elsaie ML: Photodynamic therapy in dermatology. Lasers Med Sci 24: 971-980, 2009.

15. Brown SB, Brown EA and Walker I: The present and future role of photodynamic therapy in cancer treatment. Lancet Oncol 5: 497-508, 2004.

16. Baldea I and Filip AG: Photodynamic therapy in melanoma: an update. J Physiol Pharmacol 63: 109-118, 2012.

17. Calzavara-Pinton PG, Venturini M and Sala R: Photodynamic therapy: update 2006 Part 1: Photochemistry and photobiology. J Eur Acad Dermatol Venereol 21: 293-302, 2007.

18. Kim SA, Kwon SM, Kim JA, Kang KW, Yoon JH and Ahn SG: 5'-Nitro-indirubinoxime, an indirubin derivative, suppresses metastatic ability of human head and neck cancer cells through the inhibition of Integrin $\beta 1 /$ FAK/Akt signaling. Cancer Lett 306: 197-204, 2011.

19. Jung CH, Ro SH, Cao J, Otto NM and Kim DH: mTOR regulation of autophagy. Cancer Lett 584: 1287-1295, 2010. 
20. Paglin S, Hollister T, Delohery T, Hackett N, McMahill M, Sphicas E, Domingo D and Yahalom J: A novel response of cancer cells to radiation involves autophagy and formation of acidic vesicles. Cancer Res 61: 439-444, 2001.

21. Klotz LO, Fritsch C, Briviba K, Tsacmacidis N, Schliess F and Sies H: Activation of JNK and p38 but not ERK MAP kinases in human skin cells by 5 -aminolevulinate-photodynamic therapy. Cancer Res 58: 4297-4300, 1998.

22. Wu RW, Yow CM, Wong CK and Lam YH: Photodynamic therapy (PDT): initiation of apoptosis via activation of stressactivated p38 MAPK and JNK signal pathway in $\mathrm{H} 460$ cell lines. Photodiagnosis Photodyn Ther 8: 254-263, 2011.

23. Buytaert E, Dewaele M and Agostinis P: Molecular effectors of multiple cell death pathways initiated by photodynamic therapy. Biochim Biophys Acta 1776: 86-107, 2007.

24. François A, Marchal S, Guillemin F and Bezdetnaya L: mTHPC-based photodynamic therapy induction of autophagy and apoptosis in cultured cells in relation to mitochondria and endoplasmic reticulum stress. Int J Oncol 39: 1537-1543, 2011.

25. Shinojima N, Yokoyama T, Kondo $Y$ and Kondo S: Roles of the Akt/mTOR/p70S6K and ERK1/2 signaling pathways in curcumin-induced autophagy. Autophagy 3: 635-637, 2007.
26. Iwamaru A, Kondo Y, Iwado E, Aoki H, Fujiwara K, Yokoyama T, Mills GB and Kondo S: Silencing mammalian target of rapamycin signaling by small interfering RNA enhances rapamycin-induced autophagy in malignant glioma cells. Oncogene 26: 1840-1851, 2007.

27. Kim KW, Mutter RW, Cao C, Albert JM, Freeman M, Hallahan DE and Lu B: Autophagy for cancer therapy through inhibition of pro-apoptotic proteins and mammalian target of rapamycin signaling. J Biol Chem 281: 36883-36890, 2006.

28. Levy JM and Thorburn A: Targeting autophagy during cancer therapy to improve clinical outcomes. Pharmacol Ther 131: 130-141, 2011.

29. Chen S, Rehman SK, Zhang W, Wen A, Yao L and Zhang J: Autophagy is a therapeutic target in anticancer drug resistance. Biochim Biophys Acta 1806: 220-229, 2010.

30. Choi KS: Autophagy and cancer. Exp Mol Med 44: 109-120, 2012.

31. Ahn MY, Yoon HE, Kwon SM, Lee J, Min SK, Kim TC, Ahn SG and Yoon JH: Synthesized Pheophorbide a-mediated photodynamic therapy induced apoptosis and autophagy in human oral squamous carcinoma cells. J Oral Pathol Med 43: 17-25, 2013. 